\title{
Diamond-Blackfan Syndrome. II. In Vitro Corticosteroid Effect on Erythropoiesis
}

\author{
HELEN S.L. CHAN, E. FRED SAUNDERS, AND MELVIN H. FREEDMAN ${ }^{(14)}$ \\ Division of Hematology, Hospital for Sick Children and Department of Pediatrics, University of Toronto. Toronto, \\ Ontario, Canada
}

\begin{abstract}
Summary
To study the action of corticosteroids on erythroid precursors, (burst forming unit-erythroid and colony forming unit-erythroid) in Diamond-Blackfan Syndrome (DBS), marrow from a newly diagnosed untreated infant was studied in vitro with prednisone and dexamethasone. This patient subsequently proved to be steroid responsive. Colony numbers increased linearly in an erythropoietin (EPO) dose response study. There was marked enhancement of colony numbers at all EPO doses after adding either prednisone $\left(10^{-6} \mathrm{M}\right)$ or dexamethasone $\left(10^{-9} \mathrm{M}\right)$ to the cultures. The data indicate that corticosteroids augment erythropoiesis at both early (BFU-E) and late (CFU-E) stages of development in DBS. In contrast, marrow from a second infant with DBS, clinically steroid resistant, failed to respond to steroids in vitro.

\section{Speculation}

It seems reasonable that in some patients with Diamond-Blackfan Syndrome, erythroid precursors have a relative insensitivity to erythropoietin (EPO). Corticosteroids appear able to modify EPO receptor sites on the progenitor cell membrane in vitro, thus increasing EPO sensitivity and partially correcting the erythropoietic defect.
\end{abstract}

Diamond and Blackfan described a syndrome of congenital red cell asplasia (3) (Diamond-Blackfan Anemia or Syndrome, DBS), which responds to corticosteroid therapy in the majority of patients (4). The bone marrow is initially devoid of recognizable erythroid cells; however, with treatment, it develops active erythropoiesis leading to reticulocytosis and a rise in hemoglobin. The mechanism and site of action of corticosteroids is unknown.

In most patients with DBS, marrow erythroid precursor cells, colony forming unit-erythroid (CFU-E) and burst forming uniterythroid (BFU-E), are present which are capable of replication and differentiation in tissue culture to colonies containing morphologically recognizable erythroid cells (5). Although the concentration of these progenitors has appeared to be lower than normal when studied by conventional marrow culture methods $(5,11)$ cell fractionation techniques have provided information that some patients with DBS have normal CFU-E and BFU-E numbers $(1,6)$.

To study the action of corticosteroids on erythroid precursors in the disorder, marrows from two newly-diagnosed, untreated infants with DBS were studied in vitro with prednisone and dexamethasone. The data show that these agents enhanced erythroid progenitor growth at both early and late stages of cellular differentiation in the steroid-responsive patient.

\section{MATERIALS AND METHODS}

Subjects. The infants fit the classical description of DBS (4) and are described previously (1). Patient l was considered steroid responsive whereas Patient 2 was steroid resistant. The marrows were studied in vitro before corticosteroid treatment.
Control bone marrow was obtained from two hematologically normal children aged 1 and 13 years with localized solid tumors who had marrow aspirates performed as part of their medical investigation. This study was approved by the Human Experimentation Committee of the Hospital for Sick Children and informed consent obtained.

Erythroid colony cultures (assay for BFU-E and CFU-E). Nucleated marrow cells were cultured in $0.8 \%$ methylcellulose, $30 \%$ fetal calf serum (Microbiological Associates, Bethesda, MD) and $1 \%$ bovine serum albumin (Sigma Chemical Co., St. Louis, MO) at a final volume of $1 \mathrm{ml}$ per culture plate (10). Human urinary erythropoietin (EPO) (National Heart Lung and Blood Institute, Bethesda, MD) of purity 93.5 units/mg protein was added to the cultures in the corticosteroid dose response studies and EPO of purity 3,742 units/mg protein was used in the EPO dose response study. In all studies $10^{5}$ marrow cells from controls and $10^{6}$ from the DBS patients were plated per culture. The cultures were incubated at $37^{\circ} \mathrm{C}$ for 7 or 14 days in air with $5 \% \mathrm{CO}_{2}$ and high humidity. Colonies were counted directly in the culture plates using an inverted microscope. An aggregate of more than 500 hemoglobinized cells or three or more erythroid subcolonies on day 14 was considered a "burst" or BFU-E colony (8). A CFU-E colony was defined as a cluster of 8 or more hemoglobinized cells on day 7. All studies were performed in duplicate and the results expressed as mean colony number $/ 10^{5}$ cells plated.

Steroid studies. Dexamethasone and prednisone were dissolved in 100\% ethanol, serially diluted with alpha medium (University of Toronto, Toronto, Ontario, Canada) and added to the cultures for the duration of the incubation. To determine optimum drug doses, dose response studies were done with concentrations of dexamethasone ranging from $10^{-5}-10^{-11} \mathrm{M}$ and concentrations of prednisone from $10^{-4}-10^{-9} \mathrm{M}$. Controls without added drug or with ethanol only in concentrations from $1 \%-0.0001 \%$ were also cultured.

\section{RESULTS}

In dose response studies using cultures of two control marrows there was a maximum increase in CFU-E and BFU-E using prednisone $10^{-6 ;} \mathrm{M}$ and dexamethasone $10^{-9} \mathrm{M}$ (Fig. 1). At these drug concentrations the ethanol solvent had no effect on colony number. No colonies were seen in cultures without added EPO. The culture results using marrow from patient 1 with three EPO concentrations at optimal doses of prednisone or dexamethasone are shown in Fig. 2. There was a linear increase in colony numbers in cultures without corticosteroids. With added prednisone or dexamethasone, there was significant enhancement of colony numbers at all EPO concentrations $(P<0.001)$. However, colony numbers reached only approximately $50 \%$ of control colony numbers at 1 units $/ \mathrm{ml}$ of EPO. There was no difference in the erythropoietic stimulatory effect between the two drugs. In each experiment the corticosteroid approximately doubled the number of colonies obtained with EPO alone.

In contrast, marrow from patient 2 grew no colonies at 1.0 and 2.5 units of EPO with or without steroid. At 5 units of EPO, 2 


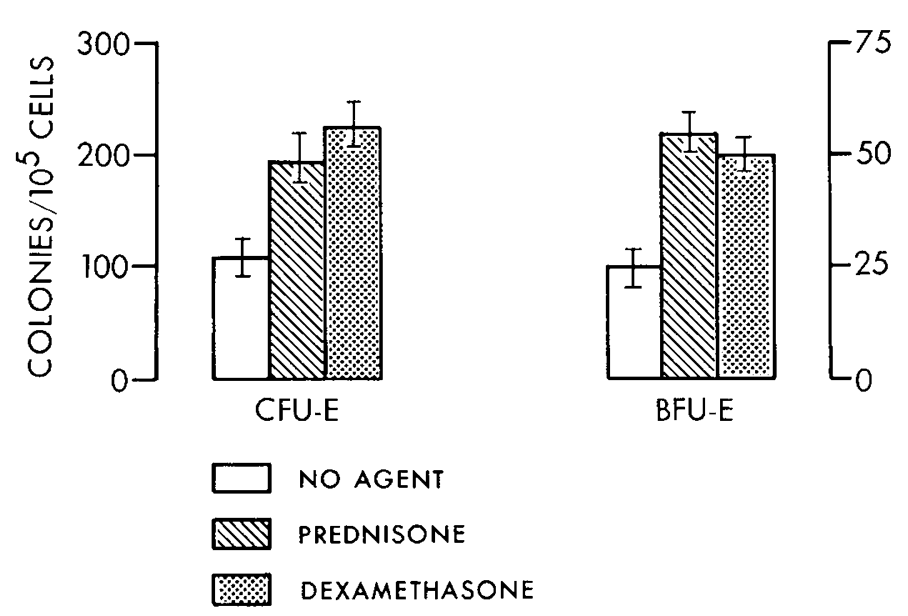

Fig. 1. The effect of added prednisone $\left(10^{-6 i} \mathrm{M}\right)$ and dexamethasone $\left(10^{-9} \mathrm{M}\right)$ on CFU-E and BFU-E colony growth from 2 control marrows using erythropoietin $1 \mathrm{unit} / \mathrm{ml}$.

CFU-E and $1 \mathrm{BFU}-\mathrm{E} / 10^{5}$ cells grew and there was no increase with either steroid.

\section{DISCUSSION}

Our data indicate that two corticosteroids, prednisone and dexamethasone, enhanced the replication and differentiation of erythroid progenitors from marrow of patient 1 in vitro at both early (BFU-E) and late (CFU-E) stages of development. Golde et al. (7) documented that dexamethasone potentiated CFU-E growth when added to cultures of fetal mouse liver, adult murine marrow, and human marrow. The mechanism of the stimulatory action was uncertain but it was only seen in cultures containing EPO. Both EPO and corticosteroids are thought to act at cell receptor sites $(2,12,13)$. Since the dexamethasone-induced erythropoietic stimulation can be abolished by progesterone (7), an agent that blocks corticosteroid activity by interfering with its binding to cell receptor sites $(12,13)$, it seems reasonable that EPO and corticosteroids could interact in some manner at cell receptor sites to produce their effect.

One can speculate that such an interaction between EPO and corticosteroids took place on the erythroid precursors of the steroid responsive patient (Patient 1) leading to a partial correction of the defect in vitro. In contrast, steroids in vitro completely failed to stimulate colony growth from the marrow of the clinically steroid resistant patient (Patient 2). The exact defect in DBS is not known but it appears to be a heterogeneous disorder both in vitro $(6,9)$ and clinically.

\section{REFERENCES AND NOTES}

1. Chan, H. S. L.. Saunders. E. F., and Freedman, M. H.: Diamond-Blackfan Syndrome 1: Erythropoiesis in Prednisone Responsive and Resistant Disease Pediatr. Res. (in press)

2. Chang, S. C., Sikkema. D., and Goldwasser, E.: Evidence for an erythropoietin receptor protein on rat bone marrow cells. Biochem. Biophy. Res. Commun., 57: 399 (1974).

3. Diamond, L. K. and Blackfan. K. D.: Hypoplastic anemia. Amer. J. Dis. Child.. 56: 464 (1938).

4. Diamond, L. K., Wang, W. C., and Alter, B. P.: Congenital hypoplastic anemia. Adv. Pediatr., 22: 349 (1976).

5. Freedman, M. H.. Amato. D., and Saunders, E. F.: Erythroid colony growth in congenital hypoplastic anemia. J. Clin. Invest., 57: 673 (1976).

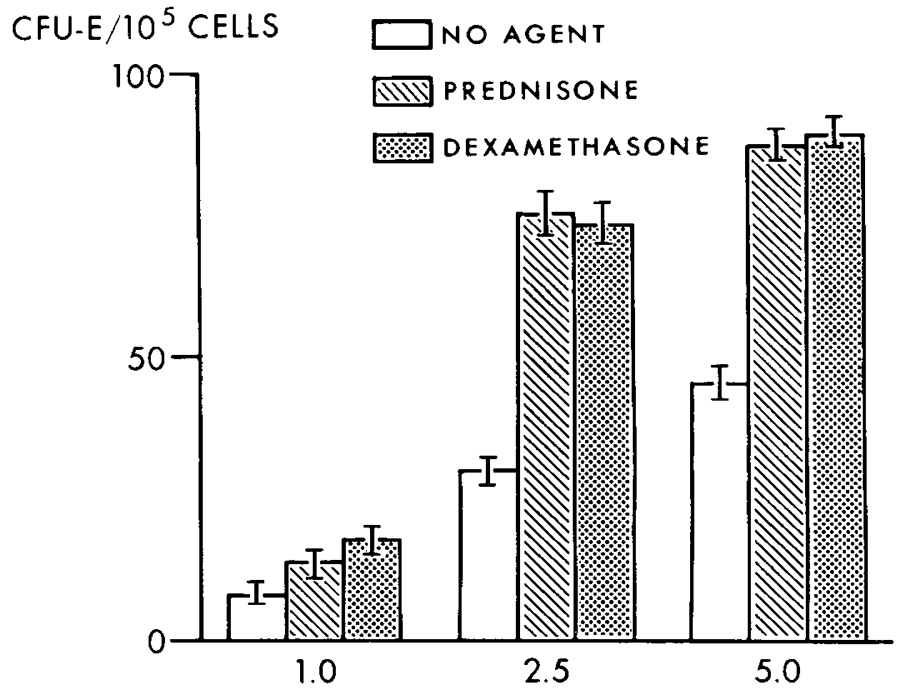

BFU-E $/ 10^{5}$ CELLS

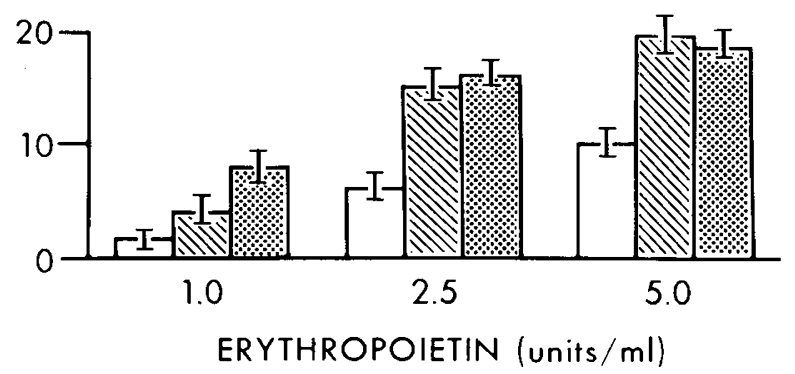

Fig. 2. The effect of added prednisone $\left(10^{-6 i} \mathrm{M}\right)$ and dexamethasone $\left(10^{-4} \mathrm{M}\right)$ on erythropoietin-stimulated CFU-E and BFU-E colony growth from marrow of a patient with Diamond-Blackfan Syndrome.

6. Freedman, M. H.. Chan. H. S. L., and Saunders, E. F.: Diamond-Blackfan syndrome: a hereditary abnormality of erythropoiesis with variable erythropoietin insensitivity. Exper. Hematol.. 7(Supp. 6): 145 (1979).

7. Golde, D. W.. Bersch, N., and Cline, M. L.: Potentiation of erythropoiesis in vitro by dexamethasone. J. Clin. Invest.. 57: 57 (1976).

8. Gregory, C. J. and Eaves, A. C.: Human marrow cells capable of erythropoietic differentiation in vitro: definition of three erythroid colony responses. Blood, 49: 855 (1977).

9. Hoffman. R.. Zanjani. E. D.. Vila, J.. Zalusky, R., Lutton. J. D.. and Wasserman, L. R.: Diamond-Blackfan Syndrome: Lymphocyte-mediated suppression of erythropoiesis. Science, 193: 899 (1976).

10. Iscove, N. N., Sieber, F., and Winterhalter. K. H.: Erythroid colony formation in cultures of mouse and human marrow: analysis of the requirement for erythropoietin by gel filtration and affinity chromatography on agarose-concanavalin A. J. Cell. Physiol.. 8.3: 309 (1974).

11. Nathan. D. G.. Clarke. B. J., Hillman. D. G.. Alter. B. P., and Housman. D. E. Erythroid precursors in congenital hypoplastic (Diamond-Blackfan) anemia. J. Clin. Invest., 61: 489 (1978).

12. Rousseau. G. G., Baxter. J. D.. Higgins, S. J., and Tomkins. G. M.: Steroidinduced nuclear binding of glucocorticoid receptors in intact hepatoma cells. J. Mol. Biol., 79: 539 (1973).

13. Rousseau, G. G.. Baxter, J. D., and Tomkins, G. M.: Glucocorticoid receptors: relations between steroid binding and biological effects. J. Mol. Biol.. 67: 99 (1972).

14. Requests for reprints should be addressed to: M.H. Freedman. M.D. Division of Hematology, Hospital for Sick Children. 555 University Ave.. Toronto, Ontario. Canada. M5G IX8.

15. Supported by Medical Research Council of Canada Grant MA-4982 and by Physicians Services Incorporated Foundation.

16. Received for publication June 11. 1981.

17. Accepted for publication November 10, 1981. 